\title{
A kinetic BGK edge-based scheme including vibrational and electronic energy modes for High-Mach flows
}

\author{
M. Fossati*, A. Mogavero, J.Herrera-Montojo \\ Aerospace Centre of Excellence, University of Strathclyde \\ 75 Montrose street, Glasgow, United Kingdom \\ J.B. Scoggins \\ CMAP, École Polytechnique \\ Route de Saclay, 91128 Palaiseau, France \\ T. Magin \\ Von Karman Institute for Fluid Dynamics \\ Chaussée de Waterloo, 72 B-1640 Rhode-St-Gense Belgium
}

\begin{abstract}
A first principles formulation for the calorically imperfect behaviour of gases is here proposed within a Boltzmann-type discretisation of the Navier-Stokes equations. The formulation is intended to enhance the consistency of gas kinetic schemes (GKS) with the physics of supersonic and hypersonic regimes where vibrational and electronic energy modes are activated before any thermal nonequilibrium or chemical activity takes place. The so-called node-pair BGK scheme, an edge-based implementation of the GKS, is considered in the present work for the implementation of a thermodynamic model where the calorically imperfect behavior is obtained from a modification of the way the different moments of the particle distribution function are computed and eventually used to determine the fluxes of conserved quantities across the boundary of each control volume. The method is validated on a series of canonical test cases for super-
\end{abstract}

\footnotetext{
* Corresponding author

Email address: marco.fossati@strath.ac.uk (M. Fossati)
} 
sonic and hypersonic flows.

Keywords: Calorically imperfect gas, GKS formulation, supersonic flow physics.

\section{Introduction}

The development of numerical schemes explicitly incorporating elements of the kinetic theory of gases is driven by the interest in formulating models directly from first principles such that assumptions on the behavior of the gas could be

5 kept to a minimum. Methods derived from the fundamental Boltzmann equation are of particular relevance to the CFD community and applications thanks to their superior modeling capabilities. Different approaches of this kind have been proposed to address flows in both incompressible and compressible regimes and flows in conditions where the classical Navier-Stokes Fourier (NSF) model might loose validity. This landscape includes particle-based methods [1, 2] and extended or generalized hydrodynamics methods $[3,4,5,6,7,8,8$. The majority of these methods was developed with the scope of addressing specific regimes and/or problems, like for example to study rarefied regimes and/or resolving accurately Knudsen layers, and might not be suitable to address in a cohesive and unified manner a wide range of flow conditions due either to their excessive computational cost (e.g. DSMC in continuum and near-continuum regimes) or loss of consistency with the laws of thermodynamics (e.g. Burnett models or Lattice-Boltzmann for high-Mach regimes) [9, 10, 6].

Among the methods aiming instead for a unified formulation capable to address a wide range of regimes (from nearly incompressible to compressible, from continuum up to continuum transitional and even rarefied regimes) while retaining a favourable computational cost, the gas kinetic scheme (GKS) originally 
proposed by $\mathrm{Xu}$ and collaborators a few decades ago [1] is a notable example.

25 The GKS scheme is obtained by adopting a simplified model of the Boltzmann's collision term proposed by Bathnagar, Gross and Krook [12] such that the solution of the Riemann problem at the cell interface, needed to compute the fluxes of the conserved quantities, can be computed in an analytical manner [13. Through the years, quite a lot of efforts have been put forward to enhance so the modeling ability of the GKS scheme to address both continuum [14] and rarefied regimes [15, high-temperature effects in the form of multiple temperatures [16, 17] and multi-species flows [18, rarefaction effects [19, 20]. From the numerical point of view, advances have been proposed to address genuinely the two and three space dimensions in a hybrid FE-FV approach [21] and in terms of higher-order discretisations based either on Finite Volumes or Discontinuous Galerkin methods [22, 23, 24].

The modeling framework provided by the GKS scheme is particularly favorable for the simulation of supersonic and hypersonic regimes and for those cases where methods adopting phenomenological approaches might not always provide sufficient accuracy. This includes for example the study of the viscous shock wave structure or the analysis of shock layers and the consequent insight that can be obtained by studying numerically potentially complex shock interaction patterns including the interference with boundary layers. From a numerical point 45 of view, the method is quite compact since no distinct discretisation for convective and viscous terms are needed, and properties like positivity preservation, intrinsic upwinding and the absence of any carbuncle effect are natively incorporated in the GKS scheme [13, 25]. The adoption of an edge-based approach bears a series of advantages, the most relevant being: the unified treatment and data structure valid for any typology of mesh in any spatial dimension and, 
in the case of the adopted node-pair formulation, the ability to have a unified treatment of both Finite Volume and Finite Element discretisation approaches [26].

From the physical modeling point of view, an aspect that, to the best of the authors' knowledge, has not been extensively addressed in the literature is the ability to reproduce from first principles the behavior of a thermally perfect but calorically imperfect gas. This behaviour emerges well before the onset of any high-temperature effects and consists in the storage of energy (also referred to 60 as activation) over the various internal modes of the gas molecules/atoms like electronic, translational, rotational, vibrational (the last two existing only in the case of polyatomic gases) 27. The macroscopic evidence of such a behaviour is that the specific heats, and their ratio $\gamma$, are no longer constant, but depend on the temperature of the gas. From an aerothermodynamics point of view this circumstance affects the overall shock wave pattern and interference process and its evolution and interaction with solid walls. In the specific case of air, the activation of the internal modes of energy occur as soon as temperature exceeds $450 \mathrm{~K}$ and modeling such a mechanism is quite important to avoid erroneous predictions of aerodynamic and thermal loads.

70

A common practice adopted to address thermally perfect, but calorically imperfect, gases in the continuum regime and before any high-temperature effects is established is to rely on available polynomial curve fits that can capture the variation of specific heats with temperature 28. Some recent interesting works 75 considering this thermodynamic behaviour are those of Li and collaborators [29] and Jiang and collaborators [30. Notably, in the first case, a GKS scheme was considered for the discretisation of the conservation equations but the formula- 
tion was presented only for the Euler equations.

The present work aims at introducing the calorically imperfect behaviour natively by means of first principles and not relying on existing curve fits in the context of a recently proposed GKS scheme [21, referred to as node-pair GKS (NPGKS). The goal is to extend the modeling capability and physical consistency of the NPGKS in addressing flows of polyatomic gases where, as a consequence of the activation of vibrational and electronic degrees of freedom, the specific heats (both at constant pressure and volume) are no longer constant but depend on the temperature. The present method is formulated under the assumption of frozen chemistry and thermal equilibrium. The former is a condition where no chemical reaction takes place but still the electrons in the atoms' orbits and in the chemical bonds can move over the different quantized levels when energy is exchanged with other molecules during the collision process. The thermal equilibrium assumption instead is to be intended as a condition where any macroscopic change of energy leads to an instantaneous redistribution of energy internally to the molecules among the different modes according to the new amount of energy in a certain location of the domain at a certain instant of time.

The manuscript is arranged as follows: in Section 2 the base node-pair GKS is briefly presented. Section 3 introduces the kinetic formulation for the calorically imperfect gas. Eventually, Section 4 reports a series of numerical experiments for supersonic flows characterizing the shock interference pattern in the case of canonical geometries like a double wedge, a double cone and in the case of an oblique shock impinging on a cylinder. 


\section{An edge-based GKS scheme}

The present section will briefly outline the adopted edge-based GKS scheme.

105

A more detailed derivation and explanation of the method, including for example the treatment of fluids with Prandtl different than one, can be found in the literature [13, 21]. Let us consider the classical Finite Volume integral form for the conservation of mass, momentum, and total energy per unit volume $\mathrm{w}=\{\rho, \rho \vec{u}, \rho e\}$

$$
\frac{d}{d t} \int_{\mathcal{C}_{i}} \mathrm{w}+\int_{\partial \mathcal{C}_{i}} \mathbf{j} \cdot \mathbf{n}=0 \quad \forall \mathcal{C}_{i} \subseteq \Omega
$$

For each dual control volume $\mathcal{C}_{i}$ in $\Omega$, the fluxes integral can be rewritten in terms of the contribution to the fluxes coming from each node $k$ connected to the central node $i$. The fluxes across the boundary of $\mathcal{C}_{i}$ can therefore be recast in terms of each $i-k$ node-pair contribution, see Figure 1

$$
\int_{\partial \mathcal{C}_{i}} \mathbf{j} \cdot \mathbf{n}=\sum_{k \in \mathcal{K}_{i, \neq}} \int_{\partial \mathcal{C}_{i k}} \mathbf{j} \cdot \mathbf{n}_{i}+\int_{\partial \mathcal{C}_{i}^{\partial}} \mathbf{j} \cdot \mathbf{n}_{i}
$$

The two terms appearing at the right hand side in equation (3) represent respectively a domain and a boundary contribution. Each term can now be approximated by means of a suitable numerical flux function, i.e. respectively $\mathbf{J}_{i k}$ and $\overline{\mathbf{J}}_{i}^{e}$

$$
\int_{\partial \mathcal{C}_{i}} \mathbf{j} \cdot \mathbf{n} \simeq \sum_{k \in \mathcal{K}_{i, \neq}} \mathbf{J}_{i k} \cdot \boldsymbol{\eta}_{i k}+\sum_{e \in \mathcal{E}_{i}^{\partial}} \overline{\mathbf{J}}_{i}^{e} \cdot \boldsymbol{\nu}_{i}^{\partial, e}
$$

where $\mathbf{j}$ represents the fluxes of conserved quantities, $\mathcal{K}_{i, \neq}$ is the set of nodes adjacent to the node $i$ and $\mathbf{J}_{i k}=\mathbf{J}\left(\mathrm{w}_{i}, \mathrm{w}_{k}\right)$ is an integrated numerical flux function at the $k$-th interface between nodes $i$ and $k . \overline{\mathbf{J}}_{i}^{e}$ is a suitable numerical flux function at the boundary, $\mathcal{E}_{i}^{\partial}$ is the set of elements of the boundary grid (in $\mathbb{R}^{d-1}$ ) defined as the intersection of the interface with the boundary of the domain $\partial \Omega . \boldsymbol{\eta}_{i k}$ and $\boldsymbol{\nu}_{i}^{\partial, e}$ are the integrated normal vectors at the interface be- 

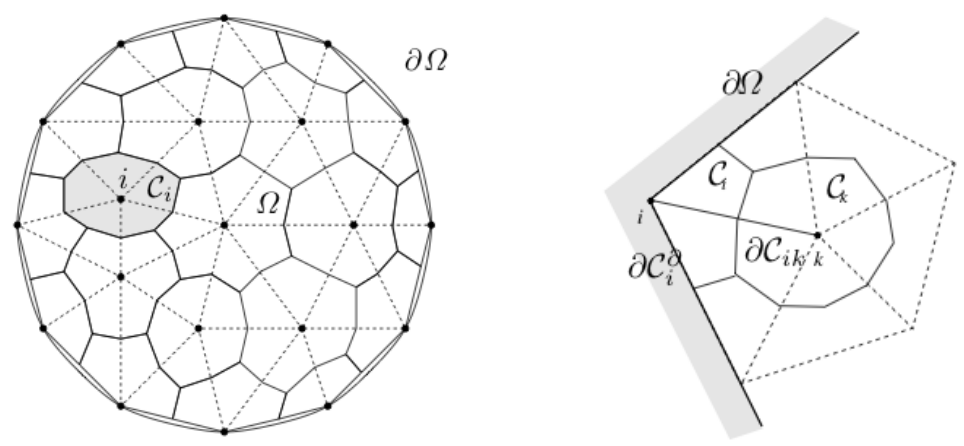

Figure 1: Node pair structure for domain nodes in red and for boundary nodes in blue (left). Boundary treatment (center and right).

tween nodes $i$ and $k$, Figure 1. A local reference frame having the $\mathrm{x}$ axis aligned with the integrated normal $\boldsymbol{\eta}_{i k}$ is introduced to explicitly identify the component of the vector of the fluxes along the direction of the integrated normal. The $k$-th domain term can then be written as follows

$$
\mathbf{J}_{i k} \cdot \boldsymbol{\eta}_{i k}=\mathrm{R}_{i k}^{-1}\left(\mathbf{J}_{i k}^{R} \cdot \boldsymbol{\eta}_{i k}^{R}\right)
$$

being $\mathrm{R}_{i k}$ the rotation matrix, $\mathbf{J}_{i k}^{R}$ the hypervector of the fluxes in the local frame and $\boldsymbol{\eta}_{i k}^{R}$ the integrated normal in the rotated frame. It is then possible to rewrite the right hand side of equation (4)

$$
\mathrm{R}_{i k}^{-1}\left(\mathbf{J}_{i k}^{R} \cdot \boldsymbol{\eta}_{i k}^{R}\right)=\mathrm{R}_{i k}^{-1}\left(\mathbf{J}_{i k}^{R} \cdot \hat{\boldsymbol{i}}_{\times}^{R}\left|\boldsymbol{\eta}_{i k}\right|\right)=\mathrm{R}_{i k}^{-1} \mathrm{~J}_{\times, i k}^{R}\left|\boldsymbol{\eta}_{i k}\right|
$$

being $J_{\times, i k}^{R}=\mathbf{J}_{i k}^{R} \cdot \hat{\boldsymbol{i}}_{\mathrm{x}}^{R}$ the desired component of the interface fluxes aligned with the normal $\boldsymbol{\eta}_{i k}$. For ease of notation the shortcut notation $\mathrm{J}_{\mathrm{x}}$ will be used to express the $k$-th flux $\mathrm{J}_{\mathrm{x}, i k}^{R}$. Similarly to the domain term, the component of the boundary fluxes aligned with the integrated normal $\boldsymbol{\nu}_{i}^{\partial, e}$ can be made explicit from each $e$-th term $\overline{\mathbf{J}}_{i}^{e} \cdot \boldsymbol{\nu}_{i}^{\partial, e}$ by introducing a local frame with the $\mathrm{x}$ axis 

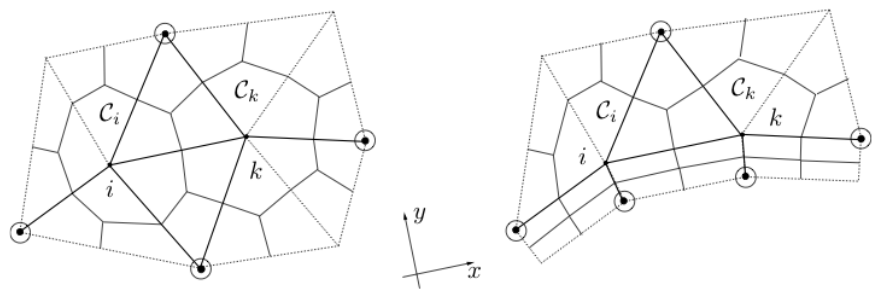

Figure 2: Extended node-pairs for the second for the gradient computation in the MUSCL approach.

aligned with the integrated normal $\boldsymbol{\nu}_{i}^{\partial, e}$

$$
\overline{\mathbf{J}}_{i}^{e} \cdot \boldsymbol{\nu}_{i}^{\partial, e}=\mathrm{R}_{e}^{-1} \bar{J}_{\mathrm{x}, e}^{R}\left|\boldsymbol{\nu}_{i}^{\partial, e}\right|
$$

where $\mathrm{R}_{e}$ is the boundary rotation matrix at node $i$. Eventually, the semidiscrete form of the conservation equations is obtained by cell-averaging over each cell $\mathcal{C}_{i}$ and by using (5) and (6) for the integrated fluxes

$$
\left|\mathcal{C}_{i}\right| \frac{d \mathrm{w}_{i}}{d t}=-\sum_{k \in \mathcal{K}_{i, \neq}} \mathrm{R}_{i k}^{-1} \mathrm{~J}_{\times, i k}^{R}\left|\boldsymbol{\eta}_{i k}\right|-\sum_{e \in \mathcal{E}_{i}^{\partial}} \mathrm{R}_{e}^{-1} \bar{J}_{\mathrm{x}, e}^{R}\left|\boldsymbol{\nu}_{i}^{\partial, e}\right|
$$

Equation (7) is valid whatever the topology of the grid and any information about the type of the elements of the geometric discretisation is enclosed in the two vectors $\boldsymbol{\eta}_{i k}$ and $\boldsymbol{\nu}_{i}^{\partial, e}$, that are referred to as metric coefficients 31, 32. Eventually, in the case of the proposed scheme, second order accuracy in space is achieved by adopting a standard MUSCL approach with Van Leer limiter. Gradients are computed by means of a Finite Difference approach based on an extended stencil associated to each single node-pair $i-k$ considering the extended nodes that are most aligned with the direction of the integrated normals, i.e. $\boldsymbol{\eta}_{i k}$ for the domain term and $\boldsymbol{\nu}_{i}^{\partial, e}$ for the boundary term [21], see Figure 2. 
The node-pair GKS scheme is obtained by resorting to the kinetic theory of gases for the explicit computation of fluxes. The gas-kinetic scheme at the basis of the present formulation is the one presented in 21]. Given the fundamental particle distribution function $f=f(\boldsymbol{x}, t, \boldsymbol{u}, \boldsymbol{\xi})$, we get

$$
\int(\boldsymbol{u} \cdot \mathbf{n}) \boldsymbol{\psi} f d \boldsymbol{u} d \boldsymbol{\xi}
$$
nodes $i$ and $k$

$$
\left\{\begin{array}{l}
\frac{\partial f}{\partial t}+\boldsymbol{u} \cdot \nabla f=\frac{1}{\tau}\left(f_{0}-f\right) \\
f(\mathbf{x}, 0, \boldsymbol{u}, \boldsymbol{\xi})= \begin{cases}f_{\mathrm{L}}(\mathbf{x}, \boldsymbol{u}, \boldsymbol{\xi}) & \mathrm{x}<0 \\
f_{\mathrm{R}}(\mathbf{x}, \boldsymbol{u}, \boldsymbol{\xi}) & \mathrm{x}>0\end{cases}
\end{array}\right.
$$

being $\times$ the direction of the integrated normal $\boldsymbol{\eta}_{i k}$ in the case of the domain integral and the direction of $\boldsymbol{\nu}_{i}^{\partial, e}$ in the case of the boundary term. In equation (9), $f_{0}$ represents the Maxwellian equilibrium function to which the gas tends 165 as a result of molecules collisions and $\tau$ indicates the characteristic time of such relaxation process. The Maxwellian state is defined as

$$
f_{0}=\rho\left(\frac{\vartheta}{\pi}\right)^{\frac{K+3}{2}} \epsilon^{-\vartheta[(\boldsymbol{u}-\boldsymbol{U}) \cdot(\boldsymbol{u}-\boldsymbol{U})+\boldsymbol{\xi} \cdot \boldsymbol{\xi}]}
$$


where $\rho$ and $\boldsymbol{U}$ are the macroscopic density and velocity, $\vartheta$ is a function of temperature, molecule mass and Boltzmann constant $\kappa$, and $\mathrm{K}$ is the dimension of the vector $\boldsymbol{\xi}$, that is the number of thermal degrees of freedom of the molecules. By means of this definition, it is possible to connect the macroscopic conserved quantities and their gradients to the particle distribution function and its derivatives. This correspondence is exploited for the actual computation of the numerical fluxes in the MUSCL approach 20]. The functions $f_{\mathrm{L}}$ and $f_{\mathrm{R}}$ that define the initial condition of the Riemann problem represent the particle 175 functions at the two nodes of the pair. These are computed by resorting to the Chapman and Enskog expansion truncated at the first order in $\tau$ computed in terms of the reconstructed macroscopic states at the two sides of the interface 34, 13, 35. The full analytical integral solution for this problem is adopted the compute the interface fluxes $[13,25$.

$$
f(\mathbf{0}, t, \boldsymbol{u}, \boldsymbol{\xi})=\frac{1}{\tau} \int_{0}^{t} f_{0}\left(\boldsymbol{x}^{\prime}, t^{\prime}, \boldsymbol{u}, \boldsymbol{\xi}\right) \mathrm{e}^{-\left(t-t^{\prime}\right) / \tau} \mathrm{d} t^{\prime}+\mathrm{e}^{-t / \tau} f(-\boldsymbol{u} t, 0, \boldsymbol{u}, \boldsymbol{\xi})
$$

For a viscous flow, the fluxes of conserved quantities locally depend on both the conserved variables and on their gradient. The Navier-Stokes equations are obtained from the BGK model by means of the Chapman-Enskog expansion 
up to first order terms in the mean collision time 36. The Maxwellian at the interface state reads

$$
f_{0}=f_{0}^{\mathrm{I}}\left[1+\mathrm{K}(x) \bar{a}_{\mathrm{L}} x+\mathrm{H}(x) \bar{a}_{\mathrm{R}} x+\bar{b} y+A t\right]
$$

where $\bar{a}_{\mathrm{L}}, \bar{a}_{\mathrm{R}}$ and $\bar{b}$ are the first order derivatives of the Maxwellian at the interface state and the coefficient $\bar{A}$ represents the coefficient associated to the first order time derivative of the Maxwellian and $H(x)$ and $K(x)$ are Heaviside functions at the left and right sides of the interface. The distribution functions for the initial state account for the first nonequlibrium term in the ChapmanEnskog expansion and can be written as follows

$$
\begin{aligned}
& f_{\mathrm{L}}=f_{0}^{\mathrm{L}}\left[1+a_{\mathrm{L}} x+b_{\mathrm{L}} y-\tau\left(a_{\mathrm{L}} u+b_{\mathrm{L}} v+A_{\mathrm{L}}\right)\right] \\
& f_{\mathrm{R}}=f_{0}^{\mathrm{R}}\left[1+a_{\mathrm{R}} x+b_{\mathrm{R}} y-\tau\left(a_{\mathrm{R}} u+b_{\mathrm{R}} v+A_{\mathrm{R}}\right)\right]
\end{aligned}
$$

being $a_{\mathrm{L}}, b_{\mathrm{L}}$, and $a_{\mathrm{R}}, b_{\mathrm{R}}$ the first order spatial derivatives of the Maxwellian distribution function, respectively at the left and right side of the interface and $A_{\mathrm{L}}$ and $A_{\mathrm{R}}$ the coefficients for the time derivatives of the Maxwellian [13. Substituting the two above definitions in the general form for the solution of the Riemann problem $[9]$, the interface distribution function giving the Navier- 
Stokes fluxes reads

$$
\begin{aligned}
f_{i k}(t) & =\left(1-\mathrm{e}^{-t / \tau}\right) f_{0}^{\mathrm{I}} \\
& +\left(t \mathrm{e}^{-t / \tau}-\tau\left(1-\mathrm{e}^{-t / \tau}\right)\right)\left[\mathrm{H}(u) \bar{a}_{\mathrm{L}} u+\mathrm{K}(u) \bar{a}_{\mathrm{R}} u+\bar{b} v\right] f_{0}^{\mathrm{I}} \\
& +\tau\left(t / \tau-1+\mathrm{e}^{-t / \tau}\right) A f_{0}^{\mathrm{I}} \\
& +\mathrm{e}^{-t / \tau}\left[\left(1-u t a_{\mathrm{L}}-v t b_{\mathrm{L}}\right) \mathrm{H}(u) f_{0}^{\mathrm{L}}+\left(1-u t a_{\mathrm{R}}-v t b_{\mathrm{R}}\right) \mathrm{K}(u) f_{0}^{\mathrm{R}}\right] \\
& -\tau \mathrm{e}^{-t / \tau}\left[\left(u a_{\mathrm{L}}+v b_{\mathrm{L}}\right) \mathrm{H}(u) f_{0}^{\mathrm{L}}+\left(u a_{\mathrm{R}}+v b_{\mathrm{R}}\right) \mathrm{K}(u) f_{0}^{\mathrm{R}}\right] \\
& -\tau \mathrm{e}^{-t / \tau}\left[A_{\mathrm{L}} \mathrm{H}(u) f_{0}^{\mathrm{L}}+A_{\mathrm{R}} \mathrm{K}(u) f_{0}^{\mathrm{R}}\right]
\end{aligned}
$$

The quasi-equilibrium assumption states that the gas requires a small amount of time to relax to equilibrium. Following Chapman and Enskog [34 the mean collision time can be computed on the basis of the relation $\tau=\mu / P$, being $\mu$ the dynamic viscosity of the gas and $P$ the pressure.

\subsection{Gas kinetic boundary conditions for the NPGKS scheme}

The numerical flux at the boundary $\overline{\mathrm{J}}_{\mathrm{x}, e}^{R}$ in equation $(7)$ is the gateway to the imposition of the boundary conditions in a weak form. The boundary flux can be obtained as

$$
\overline{\mathrm{J}}_{\mathrm{x}, \mathrm{e}}^{R}=\int u \boldsymbol{\psi} f^{\partial} d \boldsymbol{u} d \boldsymbol{\xi}
$$

where $u$ is the component of the molecular velocity normal to the boundary. $f^{\partial}$ is the distribution function at the boundary and it is computed on the basis of the macroscopic state, $\mathrm{w}_{i}^{\partial, e}$, and its relative gradient, $\nabla \mathrm{w}_{i}^{\partial, e}$ determined by the type of boundary condition to be imposed. For the numerical tests presented in Section 4, three different types of boundary conditions have been considered: supersonic inflow, outflow and isothermal wall [21]. 

conditions is obtained by resorting to the classical characteristics approach. The condition along the wall is obtained by considering the following definition for $f^{\partial}$

$$
f^{\partial}= \begin{cases}f_{\text {inc }}(\boldsymbol{u} ; \boldsymbol{x}, t) & \boldsymbol{u} \cdot \boldsymbol{n}_{\mathrm{wall}}>0 \\ f_{\mathrm{ref}}\left(\boldsymbol{u}^{\prime} \rightarrow \boldsymbol{u} ; \boldsymbol{x}, t\right) & \boldsymbol{u} \cdot \boldsymbol{n}_{\mathrm{wall}}<0\end{cases}
$$

where $f_{\text {inc }}$ is the distribution function of the molecules incident to the wall and can be computed from the state of the gas at node $i$, while $f_{\text {ref }}$, the socalled scattering kernel, represent the molecules reflected by the wall and can be expressed in terms of the Maxwellian $f_{0}^{\text {wall }}$ and $f_{\text {inc }}$ as follows 33 .

$$
f_{\text {ref }}\left(\boldsymbol{u}^{\prime} \rightarrow \boldsymbol{u} ; \boldsymbol{x}, t\right)=\zeta f_{0}^{\text {wall }}+(1-\zeta) \cdot f_{\text {inc }}(\boldsymbol{u} ; \boldsymbol{x}, t)
$$

The coefficient $\zeta$ describes the tendency of the gas to accommodate to the state of the wall. Assuming that all the molecules of the gas are diffusely reflected 230 from the wall, $\zeta$ can be recast as 37 .

$$
\zeta=\frac{\rho_{i} E_{i}-\rho_{\mathrm{ref}} E_{\mathrm{ref}}}{\rho_{i} E_{i}-\rho_{\mathrm{wall}} E_{\mathrm{wall}}}
$$

where $\rho_{i} E_{i}$ is the total energy per unit volume of the molecules at the boundary node, $\rho_{\text {ref }} E_{\text {ref }}$ is the total energy associated to the reflected particles and $\rho_{\text {wall }} E_{\text {wall }}$ is the total energy of a virtual fluid associated to the wall. In the case of isothermal wall, $\zeta=1$ [33] and therefore $f_{\text {ref }}$ coincides with $f_{0}^{\text {wall }}$ which in turn can be obtained by knowing the values imposed at the wall for the temperature and enforcing the conservation of mass at the wall [21]. 


\section{Vibrational and electronic energy modes}

The behavior of the gas in terms of its caloric equation of state, emerges as part of the the assumptions made for the internal structure of the molecules of Classical physics can still be used if the vibrational and/or electronic degrees of freedom are assumed not fully excited, under the provision that $\mathrm{K}$ in $(20)$ is not an integer number but varies as a result of the energy redistribution over the available energy levels.

$$
\begin{aligned}
C_{V} & =(K+3) \frac{1}{2} \mathcal{R} \\
C_{P} & =\mathcal{R}+C_{V}
\end{aligned}
$$


A widely-used approach to address this mechanism is to address the variation of specific heat with temperature by means of polynomial fit of experimental data 28. This approach can also be applied in the case of the GKS scheme described above, by using a non integer $\mathrm{K}$ in 10$]$ [29]. However, it is evident that the definition of $\mathrm{K}$ given in $(10)$ as the dimension of the vector $\boldsymbol{\xi}$ becomes less rigorous from a mathematical point of view. Despite this observation, it can be easily proved that the internal energy obtained integrating 10 with non integer $\mathrm{K}$ is still consistent with (20). This consideration does not apply for the other moments of the Maxwellian with respect to the internal degrees of freedom of the gas (the internal energy is the moment of order 2), which are required during the calculation of the BGK fluxes, i.e.

$$
\begin{aligned}
\left\langle\boldsymbol{\xi}^{2 n}\right\rangle= & \int_{-\infty}^{\infty}(\boldsymbol{\xi} \cdot \boldsymbol{\xi})^{n} f_{0}(\boldsymbol{\xi}) d \boldsymbol{\xi}= \\
& \int_{-\infty}^{\infty}(\boldsymbol{\xi} \cdot \boldsymbol{\xi})^{n} \rho\left(\frac{\vartheta}{\pi}\right)^{\frac{K+3}{2}} \epsilon^{-\vartheta[(\boldsymbol{u}-\boldsymbol{U}) \cdot(\boldsymbol{u}-\boldsymbol{U})+\boldsymbol{\xi} \cdot \boldsymbol{\xi}]} d \boldsymbol{\xi}
\end{aligned}
$$

When the Maxwellian is the same for every degree of freedom, the aforementioned moments can be calculated analytically to obtain [13, 20]

$$
\begin{aligned}
\left\langle\boldsymbol{\xi}^{0}\right\rangle & =1 \\
\left\langle\boldsymbol{\xi}^{2}\right\rangle & =\frac{K}{2 \vartheta} \\
\left\langle\boldsymbol{\xi}^{2 n}\right\rangle & =\left\langle\boldsymbol{\xi}^{2(n-1)}\right\rangle \frac{K+2(n-1)}{2 \vartheta}
\end{aligned}
$$
mally perfect gas. As anticipated above, this is consistent for $n=2$ in equation (22), since it leads back to equation 20 . However it is not rigorously correct to calculate all the moments of the Maxwellian needed to compute the high-resolution numerical fluxes [38, 39]. A more rigorous methodology is here 
280 pothesis of calorically perfect gas is lost. The methodology is based on the reconstruction of the specific heat by means of established models for the vibrational and electronic excitation contributions. This approach has the advantage of keeping the physical consistence of the kinetic flux model avoiding the need for the non-integer number of degrees of freedom approximation.

In order to calculate the moments in a general fashion, it is convenient to rewrite 21 developing the power $(\boldsymbol{\xi} \cdot \boldsymbol{\xi})^{n}$, in terms of products of its components:

$\left\langle\boldsymbol{\xi}^{4}\right\rangle=\sum_{i=1}^{K}\left\langle\xi_{i}^{4}\right\rangle+2 \sum_{i=1}^{K}\left\langle\xi_{i}^{2}\right\rangle \sum_{j=i+1}^{K}\left\langle\xi_{j}^{2}\right\rangle$

$\left\langle\xi^{6}\right\rangle=\sum_{l=1}^{K}\left\langle\xi_{l}^{6}\right\rangle+3 \sum_{l=1}^{K} \sum_{i=l+1}^{K}\left(\left\langle\xi_{l}^{2}\right\rangle\left\langle\xi_{i}^{4}\right\rangle b+\left\langle\xi_{l}^{4}\right\rangle\left\langle\xi_{i}^{2}\right\rangle\right)+6 \sum_{l=1}^{K} \sum_{i=l+1}^{K} \sum_{j=i+1}^{K}\left\langle\xi_{l}^{2}\right\rangle\left\langle\xi_{i}^{2}\right\rangle\left\langle\xi_{j}^{2}\right\rangle$

with $\left\langle\xi_{i}^{n}\right\rangle=\int_{-\infty}^{\infty} \xi_{i}^{n} f_{i}\left(\xi_{i}\right) d \xi_{i}$

\subsection{Kinetic formulation for the vibrational energy modes}

The effects of the vibrational degree of freedom have here been modeled using the harmonic oscillator theory, according to which, the internal vibrational 
energy per unit mass, $u_{v}$, can be written as 36

$$
\begin{aligned}
& u_{v}=\frac{\mathcal{R}}{Z_{v}} \sum_{j=0}^{\infty} j \Theta_{v} e^{-\frac{j \Theta_{v}}{T}} \\
& \text { where } Z_{v}=\sum_{j=0}^{\text {All states }} e^{-\frac{j \Theta_{v}}{T}}=\frac{1}{1-e^{-\frac{\Theta_{v}}{T}}}
\end{aligned}
$$

where $\Theta_{v}$ is the characteristic temperature of the harmonic oscillator, $T$ is the macroscopic temperature and $Z_{v}$ is the partition function for the quantized vibrational levels. Equation (24) is derived from the probability that a molecule has to vibrate with the $\mathrm{j}$-th harmonics

$$
f_{0}^{v}(j)=\frac{1}{Z_{v}} e^{-\frac{j \Theta_{v}}{T}}
$$

Equation 25 represents the Maxwellian distribution of a quantized degree of freedom, that as opposed to the classical limit case, is not a probability density, but a finite probability. The moment with respect to the vibrational degree of freedom, as defined in (23), is then

$$
\left\langle\xi_{v}^{2 n}\right\rangle=\frac{\mathcal{R}^{n} \Theta_{v}^{n}}{Z_{v}} \sum_{j=0}^{\text {All states }} j^{n} e^{-\frac{j \Theta_{v}}{T}}
$$

Each term in the sum of equation $(26)$ becomes smaller and smaller as $j$ increases since $j \Theta_{v}$ becomes bigger and bigger. Therefore, for a given $T$, the progressive inclusion of more terms will result in smaller and smaller changes in the value of the moment. In practice, a threshold can be defined to be used as a means to stop the calculation of more terms in the summation over all vibrational states.

In Figure 3 the specific heat calculated by means of the harmonic oscillator 

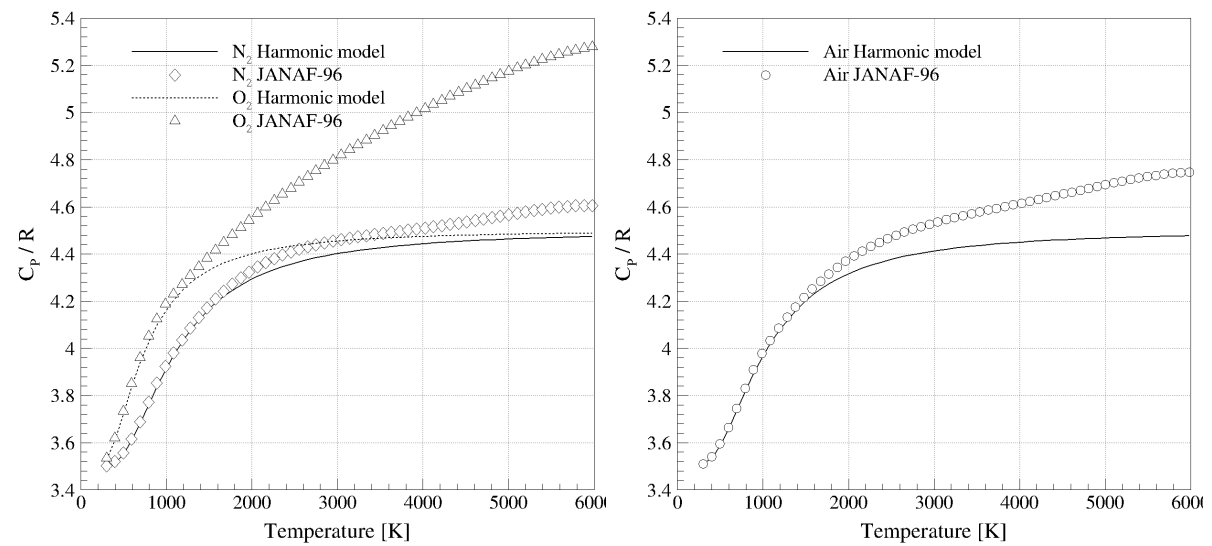

Figure 3: Specific heat as function of temperature. Comparison between experimental data and harmonic oscillator theory 28, 36.

theory is compared with a polynomial data fit 28$]$ for Nitrogen and Oxygen. As can be observed, the harmonic oscillator theory provides values for the $C_{P}$ that progressively depart from the polynomial fit. At about 3,000 K the difference between the harmonic oscillator approximation and the polynomial fit is about $2.6 \%$ for $C_{P}$ and about $3.1 \%$ for $C_{V}$ in the case of air, see Figure 3 right and Table 1. As the temperature increases, other effects need to be taken into account in order to ensure consistency with the physics. Namely, after 1,000 $\mathrm{K}$ the electronic excitation becomes not negligible for the Oxygen, while for the Nitrogen, only small departures are observed, see Figure 4. Such difference between Oxygen and Nitrogen in this case, is due to the different distributions of quantized levels, i.e. the distance between two subsequent quantized levels and the energy associated to each one of these, for the two molecules that, for the same level of temperature, allows more energy to be stored in the molecules of Oxygen. 


\begin{tabular}{c|c|c} 
& Polynomial fit & Harmonic oscillator \\
\hline$C_{P} / \mathcal{R}$ & 4.527 & 4.409 \\
$C_{V} / \mathcal{R}$ & 3.527 & 3.409
\end{tabular}

Table 1: Specific heat values from polynomial fit and harmonic oscillator theory at $3000 \mathrm{~K}$.

\subsection{Kinetic formulation for the electronic energy modes}

A similar approach can be used to take into account the contribution of electronic excitation. Thermal energy can be stored by the electrons of a molecules, that can move away from their ground state, while staying in the molecule (i.e. no dissociation) [36. Given the structure of the molecule a set of electronic states is defined by means of their characteristic temperatures $\Theta_{e}^{i}$. As a consequence the probability of electrons staying into a certain state is given by:

$$
\begin{aligned}
& f_{0}^{e}(j)=\frac{1}{Z_{e}} g_{j} e^{-\frac{\Theta_{e}^{j}}{T}} \\
& \text { where } Z_{e}=\sum_{j=0}^{\infty} g_{j} e^{-\frac{\Theta_{e}^{j}}{T}}
\end{aligned}
$$

where $g_{j}$ is the degeneracy factor, that is the number of states with characteristic temperature $\Theta_{e}^{j}$. For electrons several states can degenerate and have the same energy. Similarly to the vibration, the moment with respect to the electronic degree of freedom, as defined in 23 is then given by

$$
\left\langle\xi_{e}^{2 n}\right\rangle=\frac{\mathcal{R}^{n}}{Z_{e}} \sum_{j=0}^{N_{e}}\left(\Theta_{e}^{j}\right)^{n} e^{-\frac{\Theta_{e}^{j}}{T}}
$$

where $N_{e}$ is the total number of electronic states. In Figure 4 electronic contribution to $C_{P}$ as calculated here is added and compared against polynomial data. With reference to the case of air, the electronic contribution has a big impact for the Oxygen, while it has a negligible impact on Nitrogen. 

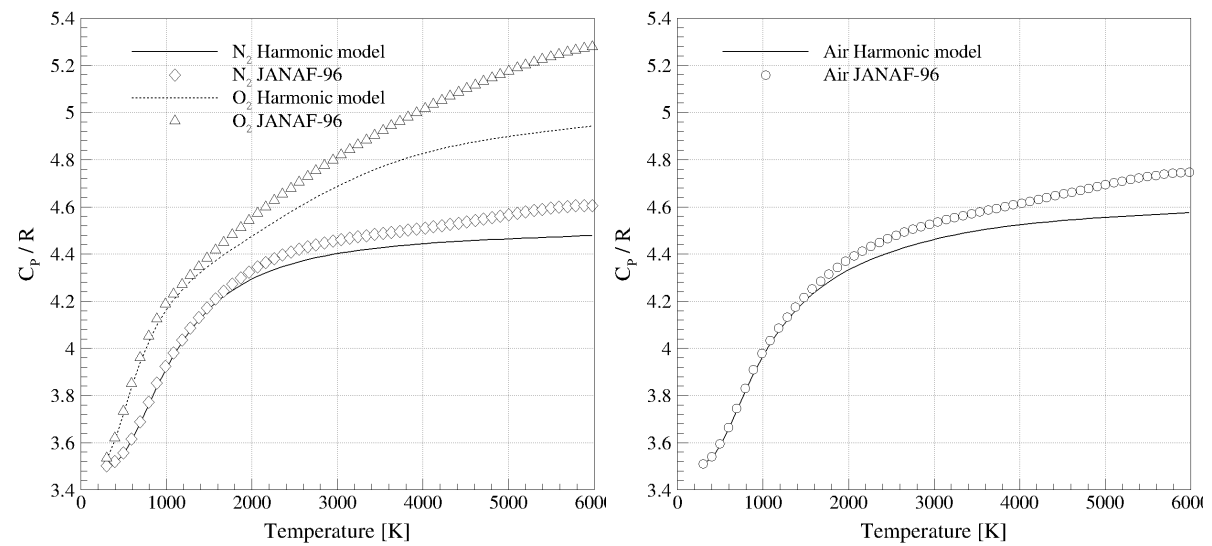

Figure 4: Specific heat as function of temperature. Comparison between experimental data and harmonic oscillator theory and electronic excitation 28,36

A residual difference between the polynomial dataset and modeling presented above remains. This can be explained by second order effects such as the coupling of the vibrational and the rotational degrees of freedom and the fact that the molecule is not a perfect harmonic oscillator [36].

\section{Numerical test cases}

The changes in the way energy can be stored in the molecules of the gas has an impact on the overall flow field and on the aerodynamic and thermal loads acting on a body interacting with a supersonic airflow and in the following a series of canonical test cases will be considered to exemplify such a condition.

The proposed NPGKS formulation has been implemented within the opensource solver SU2 [40] and modifications have been applied to the thermochemistry library Mutation ++41 in order to compute the appropriate moments as per equations (26) and (28). The former is a CFD solver that is rapidly estab-

lishing within the open-source community as a prominent software for aerospace 


\begin{tabular}{l|c}
\hline Mach & 7.11 \\
Unit Reynolds & 55,880 \\
Static temperature $[\mathrm{K}]$ & 191 \\
Static pressure $[\mathrm{Pa}]$ & 390.935 \\
Wall temperature $[\mathrm{K}]$ & 300 \\
Test time $[\mu \mathrm{s}]$ & 327 \\
\hline
\end{tabular}

Table 2: Conditions for the double wedge configuration at $30-55$ deg.

design, the latter is a well established library of thermochemical/physical properties for various gas species.

\subsection{Air flow over a double wedge $30^{\circ}-55^{\circ}$}

speeds. This configuration has been studied extensively both numerically and experimentally [42].

The case considered here is the Mach 7.11 flow of air under the conditions presented in Table 2, It has been observed that when trying to address this an unsteady behaviour characterized by flow separation induced by the impingement of shock waves on the wall. The mesh adopted is a hybrid mesh with 457,121 elements and 261,120 nodes and the spacing of the mesh has been guided by existing literature addressing the same test case 43 . The simulation was run with a dual time-stepping approach with a uniform time step in the true time equal to $1 \times 10^{-8} \mathrm{~s}$. The CFL adopted in the pseudo-time was 0.1 


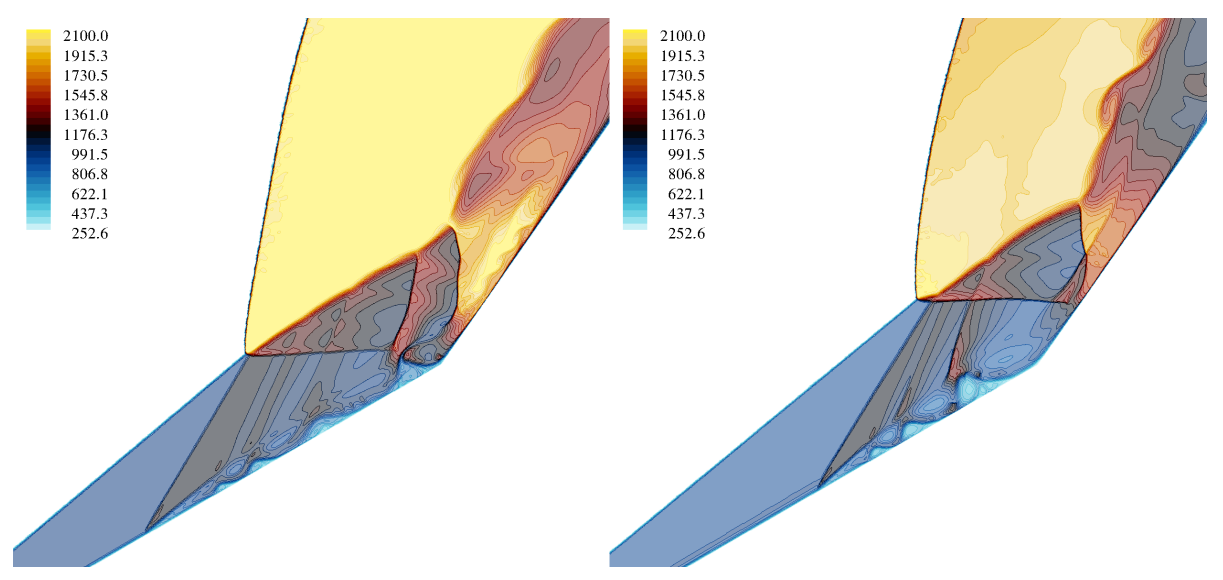

Figure 5: Double wedge configuration at $30-55 \mathrm{deg}$. Temperature field at $150 \mu \mathrm{s}$. Calorically perfect model (left) and thermally perfect model (right).

and an explicit Euler integration scheme was used. Wall time of the simulation was about 10 days on 120 cores. A detailed experimental study has been proposed by Swantek and collaborators [4] and their work is taken here as a reference case to validate the proposed approach. Following the recent work of Durna and collaborators [43], Figures 5 and 6 illustrate the flow field at $150 \mu \mathrm{s}$ in proximity of the region where the change of slope is located. A comparison is made between the flow field obtained with a calorically perfect model and a thermally perfect one. Figure 5 presents contours of temperature while Figure 6 illustrates the density gradient fields. It can be shown that assuming a calorically perfect model induces a more prominent shock-induced separation region and an anticipated location of the separation oblique shock. The shear region behind developing nearby the triple point is more complex in the case of the calorically perfect gas as a consequence of the series of shock waves generated by the recirculation region behind the separation. In both cases, the shear region develops a Kelvin-Helmholtz instability.

A visual comparison of the present thermally perfect solution with the solution presented in [43] at the same instant of time (not reproduced here but available 

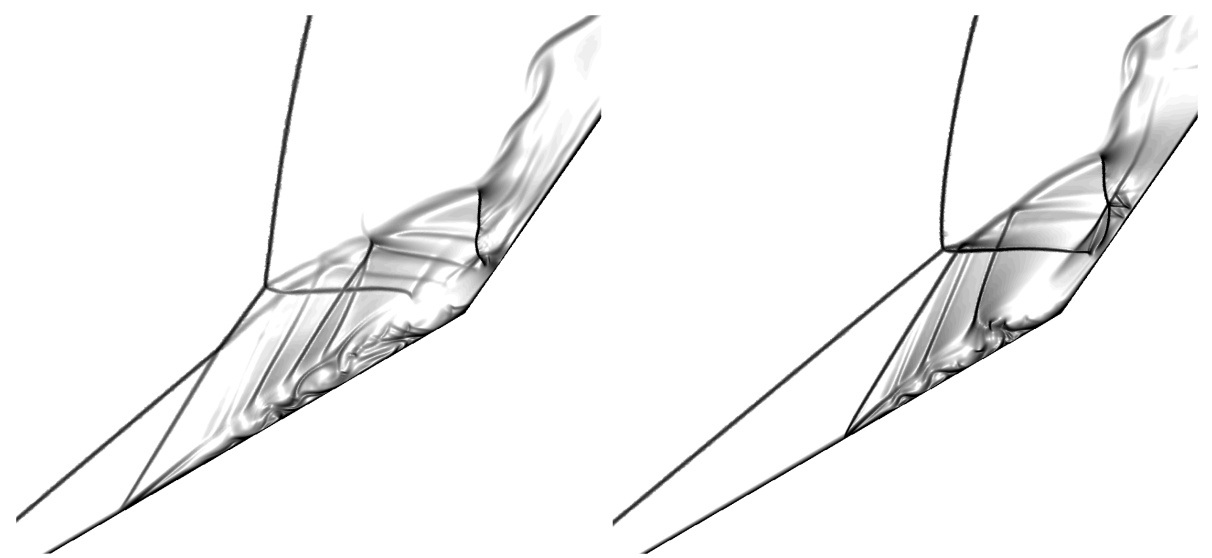

Figure 6: Double wedge configuration at $30-55 \mathrm{deg}$. Density gradient at $150 \mu \mathrm{s}$. Calorically perfect model (left) and thermally perfect model (right).

in the literature) shows quite a similar flow pattern. Note anyway that the results presented in 43 refer to a flow of Nitrogen with a thermally perfect model based on the JANAF tables. A quantitative comparison is presented instead in Figure 7 where the time-averaged profiles of normalized pressure and heat flux are presented. In the case of heat flux the experimental results of Swantek and collaborations 44 are also presented. The time averaging was realized between $150 \mu$ s and $310 \mu \mathrm{s}$ as done in [43. The agreement between the thermally perfect model and the experiments is fair and improvement with respect the case of calorically perfect gas is visible in terms of position of the heat flux peak and its magnitude. When observing the range of temperature obtained in this case, it has to be noted that a maximum value of approx. $2,100 \mathrm{~K}$ is obtained in the case of calorically perfect gas. This would result in the initiation of molecular Oxygen dissociation and a more sophisticated numerical model. In the case of a thermally perfect gas instead the temperature approaches 2,000 K and a model with no chemistry still remains acceptable. 

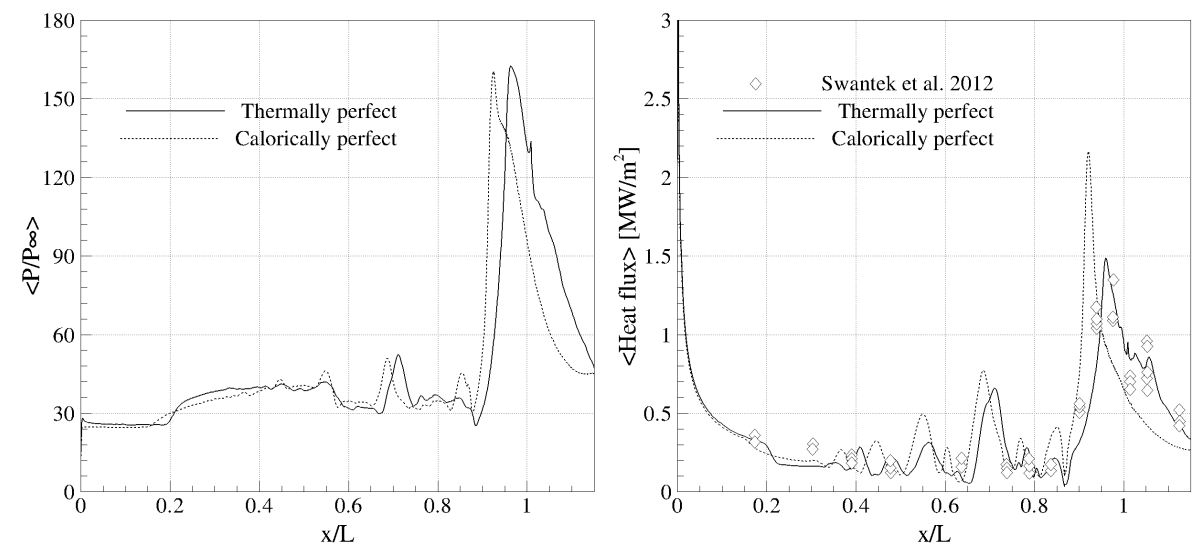

Figure 7: Double wedge configuration at $30-55$ deg. Time-averaged normalized pressure (left) and heat flux (right).

\subsection{Air flow over a double cone $25^{\circ}-50^{\circ}$}

The second test case is the Mach 8 viscous flow over a double cone configuration with the fore angle of $25^{\circ}$ and the aft angle $50^{\circ}$. The Reynolds number based on the model base diameter of $0.038 \mathrm{~m}$ is 270,000 , the freestream temperature is $57 \mathrm{~K}$ and the wall is assumed isothermal at $590 \mathrm{~K}$ [45. This is once again a canonical test case considered to test the ability of numerical schemes to predict the complex flow pattern established around the cone. Table 3 summarizes the flow conditions for this test case. The flow is assumed to be laminar despite the value of the Reynolds number might indicate a turbulent flow. Following the work of Wright and collaborators [45] it is shown that for this test case a marginal difference is observed between a laminar and turbulent flow. A 2D fully structured mesh has been used in this case consisting of 426,478 quadrilateral elements and 428,050 nodes with 350 points in the normal direction to the surface of the cone. The problem converged to a steady-state solution using a CFL equal to 0.3 with an Euler explicit integration scheme. The problem was solved using axisymmetric conditions and the wall time required to get convergence was about 7 days on 120 cores. 


\begin{tabular}{l|c}
\hline Mach & 8 \\
Unit Reynolds & 270,000 \\
Static temperature [K] & 57 \\
Static Pressure [Pa] & 359.868 \\
Wall temperature [K] & 590 \\
\hline
\end{tabular}

Table 3: Conditions for the double cone configuration at 25 - $50 \mathrm{deg}$.

Figure 8 illustrates the system of shock waves that is established that follows the classical taxonomy provided by Edney [46]. The system is characterized by a significant interaction between the shock waves and the boundary layer along the wall that induces a visible recirculation region in proximity of the region where the angle increases from $25^{\circ}$ to $50^{\circ}$ and a shear layer originating from the point where multiple shocks are converging. A sequence of reflecting shocks is generated between the shear layer, i.e. the contact discontinuity, and the wall at $50^{\circ}$. Figure 9 reports the contours of the density gradient and illustrates the establishment of a classic Edney type IV interaction in proximity of the change of slope of the cone. A reattachment compression train is observed evolving towards a reattachment shock. A clear shear layer is observed interacting with a series of reflected shocks.

A quantitative comparison is presented in Figure 10 showing a comparison between the computed pressure and heat fluxes profiles along the wall and available experimental data from Magruder [47. (only for pressure) also reported in [45. It can be observed that the adoption of a thermally perfect gas model shows good agreement with the experimental data with visible differences with respect to the calorically perfect model. The right plot presents the normalized heat fluxes along the wall and it can be observed the difference between the two models in terms of peak values of heat and also distribution. In order to better assess the difference between the two models, an integral measure of the overall heat transferred to the wall in both cases is presented in Table 4.2. A difference 


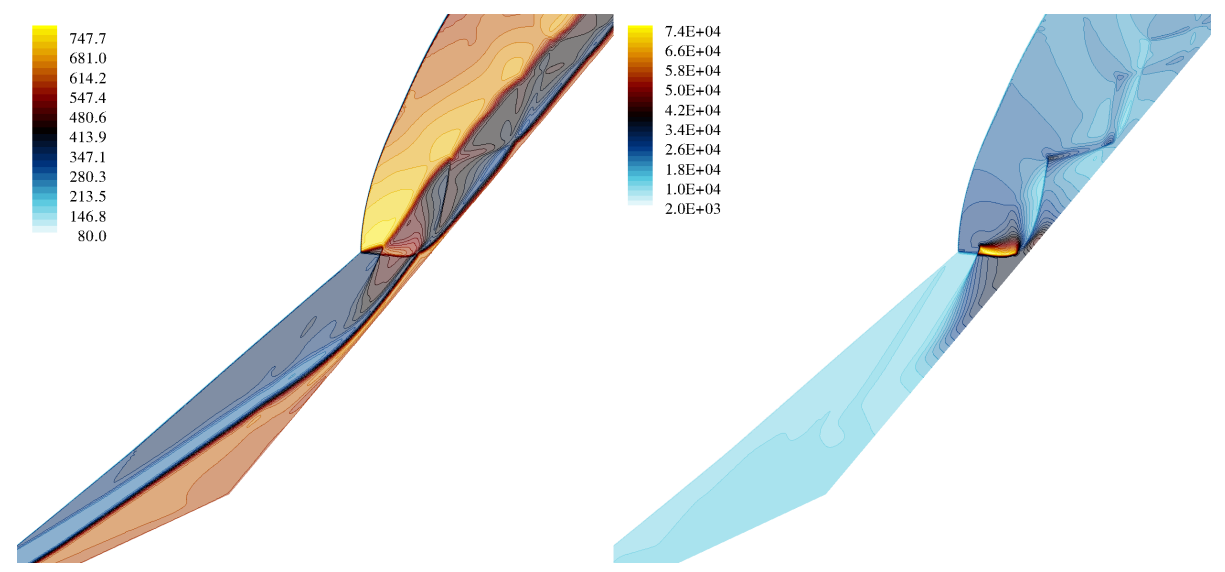

Figure 8: Double cone configuration at 25 - 50 deg. Contours of temperature [K] (left). Pressure $[\mathrm{Pa}]$ (right).

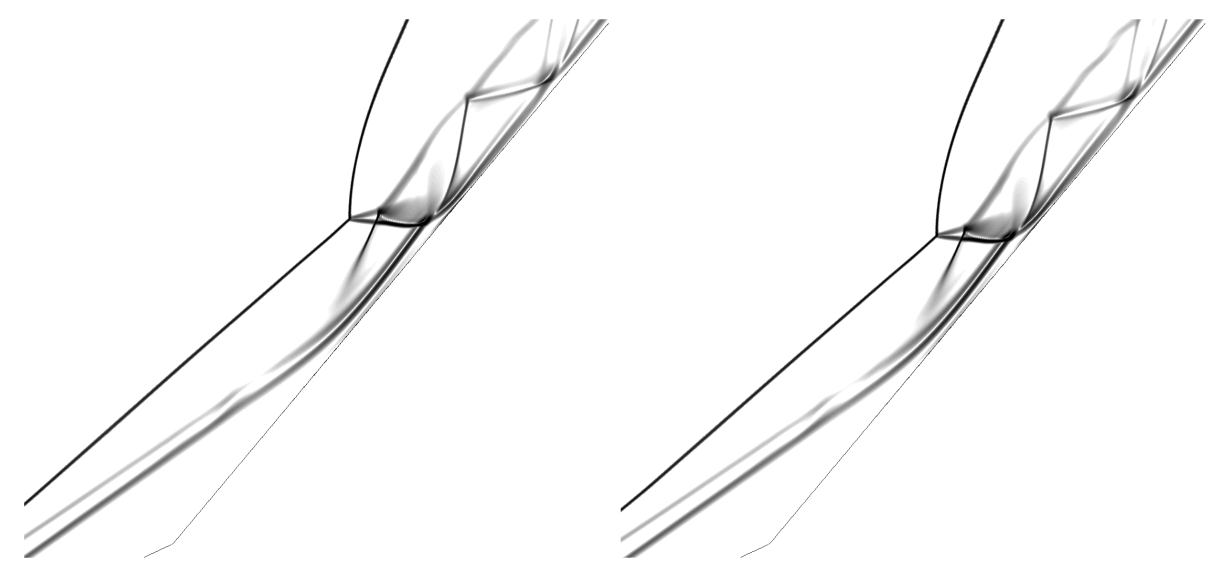

Figure 9: Double cone configuration at 25 - 50 deg. Density gradient. Calorically perfect gas (left), thermally perfect gas (right). 

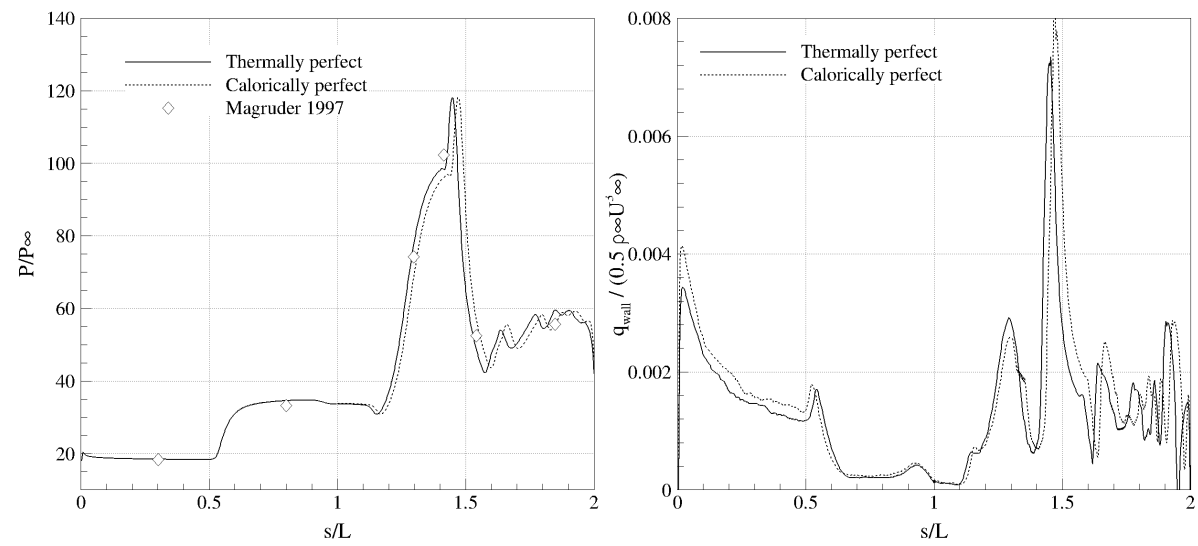

Figure 10: Double cone configuration at 25 - 50 deg. Normalized pressure profile (left), normalized heat flux (right).

\begin{tabular}{l|c|c} 
& Calorically perfect & Thermally perfect \\
\hline Heat flux $[\mathrm{W} / \mathrm{m}]$ & 700.886 & 640.717
\end{tabular}

Table 4: Heat flux per unit depth transferred from the flow to the wall $[\mathrm{W} / \mathrm{m}]$.

of about $8.6 \%$ is observed.

In this case, the temperature at the wall is assumed to be constant at $590 \mathrm{~K}$

the major differences between a calorically perfect and an imperfect one are expected inside the flow field where the angles and evolution of the shock waves pattern established in the region above the wall will be more affected given that temperature reaches a maximum of $\simeq 770 \mathrm{~K}$ in the post-shock region.

\subsection{Edney IV interference pattern over a cylinder}

Eventually, the last test case presented here is the two-dimensional supersonic flow of air over a cylinder in presence of an impinging oblique shock. This type of interaction has been subject of the study of Edney [46] who classified up to six different shock interference patterns depending on the vertical position of 
the impinging oblique shock. Among these, the type IV has been recognized as the most severe case with respect to the pressure and heat peak amplification over the case with no impinging shock. Type IV interaction has been studied extensively numerically [48] and experimentally [49] in the literature due to its representation of the most severe conditions what could occur in proximity of an engine cowling with an oblique shock generated somewhere else ahead along the vehicle. The type IV interaction is characterized by a reflected shock and a shear layer region with a supersonic jet that develops into a bow shock wave in proximity of the cylinder surface. It is the presence of this bow shock that determines the highest amplification of pressure and heat loads among the other types of interaction. Also in this case, the literature reports unsteadiness of the flow once the complete structure of the flow is established. This unsteadiness is revealed in the present work also and consists of a vertical oscillation of the supersonic jet that is connected to a series of disturbances propagating upwards and downwards from the bow shock location.

The conditions of the simulation were taken from 48 and are summarized in Tables 5 and 6 . Note that a study of this test case was also performed by means of a BGK kinetic scheme by Xu and collaborators [50] but adopting a flow of Nitrogen instead of air and assuming a calorically perfect gas. A more recent work studying numerically the same type of shock-shock interaction in the case of air have been presented by Xu and collaborators [17. In this case, a freestream Mach 10 flow of air has been considered with a GKS model accounting for thermal nonequilibrium conditions, i.e. multi-temperature models. In this case a steady-state condition is also established. It is worth noting that the inclusion of nonequilibiurm effects usually acts in the direction of promoting the establishment of a stationary field. Another relevant feature of this work was 


\begin{tabular}{l|c}
\hline Unit Reynolds & $6.75 \times 10^{6}$ \\
Wall temperature [K] & 294.44 \\
Cylinder radius [m] & 0.0381 \\
Impinging shock angle [deg] & 18.1114 \\
\hline
\end{tabular}

Table 5: Conditions for the type IV pattern over a cylinder.

\begin{tabular}{l|c|c} 
& Freestream & Behind oblique shock \\
\hline Mach & 8.03 & 5.25 \\
Temperature $[\mathrm{K}]$ & 111.56 & 238.04 \\
Pressure $[\mathrm{Pa}]$ & 985.01 & $6,996.7$ \\
Flow direction [deg] & 0 & 12.5 \\
\hline
\end{tabular}

Table 6: Conditions for the type IV pattern over a cylinder. Shock relations.

the explicit inclusion of a shock generator in the form of a wedge positioned in front of the cylinder to replicate the experimental settings. A hybrid unstructured mesh was used consisting of 224,155 elements and 136,415 nodes. A dual time-stepping second order approach was used for the unsteady simulation with a time step of $1 \times 10^{-6} \mathrm{~s}$ and a CFL in the pseudo-time of 0.1. Euler explicit integration scheme was considered. The total simulation time was $6 \times 10^{-4} \mathrm{~s}$ and the wall time for the simulation was about 4 days on 80 cores. Figures 11 and 12 illustrate the results obtained with a thermally perfect model only. Due to the unsteadiness of the flow, Figure 11 shows the contours of temperature and pressure at $550 \mu \mathrm{s}$ while Figure 12 shows the density contours for two instants of time, one at the beginning of the averaging period, i.e. $500 \mu \mathrm{s}$ and one at the end of it at $600 \mu$ s illustrating the structure of shock interference pattern of type IV. Eventually, Figure 13 provides a quantitative comparison of the results obtained with the present node-pair GKS scheme with thermally perfect thermodynamics and experimental results available in the literature [49]. The comparison is made with the time averaged results between $500 \mu \mathrm{s}$ and $600 \mu \mathrm{s}$ and in the plot the average values are shown together with the standard deviation of pressure and heat fluxes to have a representation of the boundaries of 
the variation of the profiles.

The agreement with the reference profiles is fair with respect to the location of the peak of heat flux and pressure on the surface of the cylinder and with respect to the values of the peak normalized with respect to the relative stagnation values in the case with no oblique shock [49. The undisturbed stagnation values for pressure and heat flux have been taken following the approach proposed by Zhong [48, i.e. $Q_{0}=470,501.391 \mathrm{~W} / \mathrm{m}^{2}$ and $P_{0}=83.85 P_{\infty}$. Figure 13 reveals a visible underprediction of heat flux in the region immediately below the bow shock, and the corresponding peak on the surface. A similar underprediction is also observed, even if less intense in the instantaneous profiles presented by Zhong [48] and also in the results presented by Xu [50] (even if Xu considered Nitrogen and not air as in the present and Zhong's work). A possible explanation of such a discrepancy could be referred to the high sensitivity of the wall quantities from the position of the shock and the pre- and post-shock states. In this work, in fact, no explicit shock generator has been included in the simulation and the pre- and post-shock conditions were taken from the existing literature [48. As a consequence it is possible that any discrepancy between an imposed and generated oblique shock conditions could result in a sensible difference in the shock interference patterns and in turn the pressure and heat flux profiles along the surface of the cylinder.

\section{Final remarks}

The proposed node-pair GKS scheme incorporating a first principles formulation for a thermally perfect gas has shown a fair agreement with available reference data for a series of canonical test cases where the effect of vibrational and electronic energy modes becomes sensible. The comparison of the proposed 

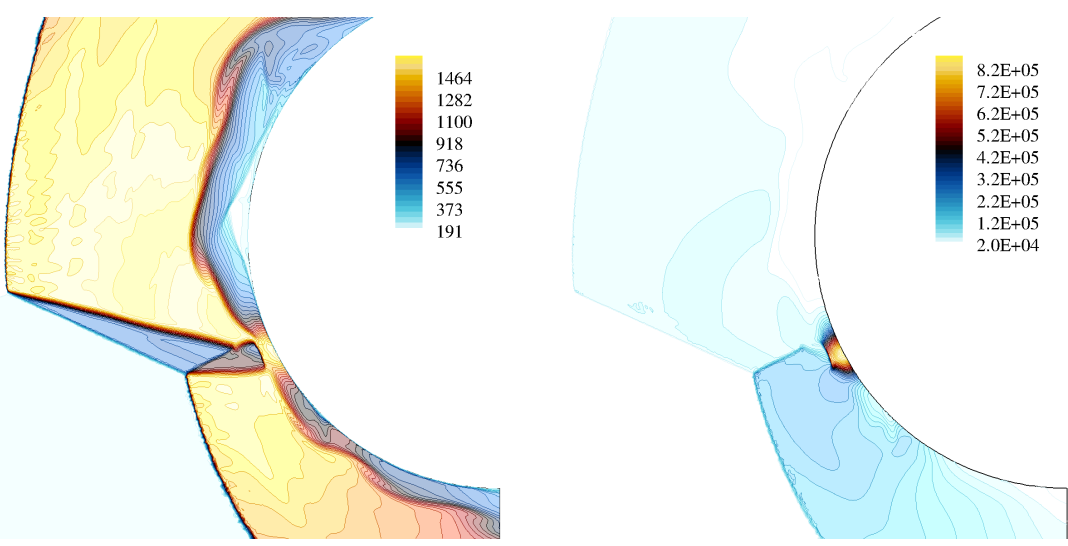

Figure 11: Edney IV pattern over a cylinder. Contours of temperature [K] (left). Pressure $[\mathrm{Pa}]$ (right). Thermally perfect gas model.
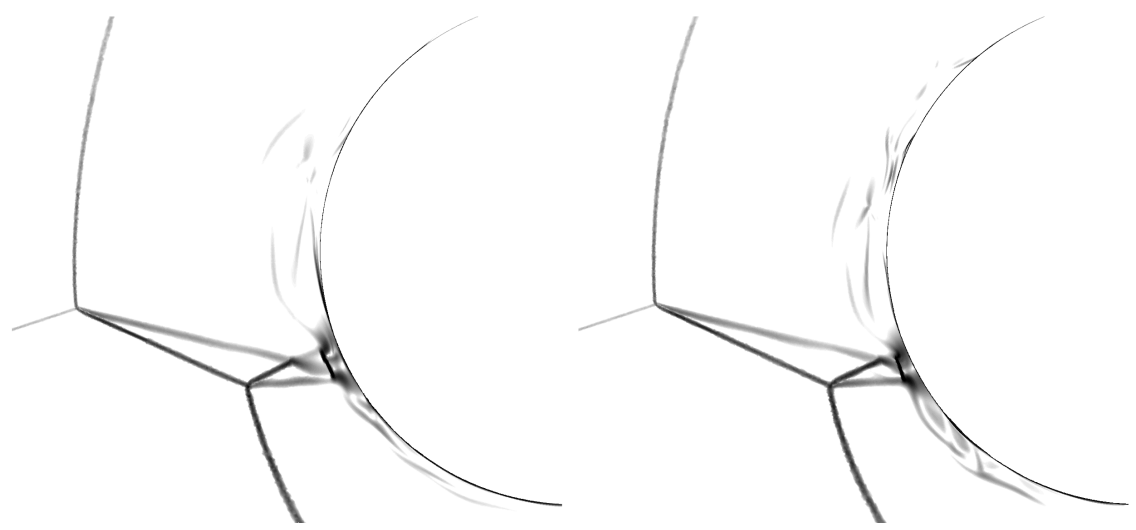

Figure 12: Edney IV pattern over a cylinder. Density gradient at two different instants of time. Beginning of the average period, $\mathrm{t}=500 \mu \mathrm{s}$ (left). End of average period, $\mathrm{t}=600 \mu \mathrm{s}$ (right). Thermally perfect gas model. 

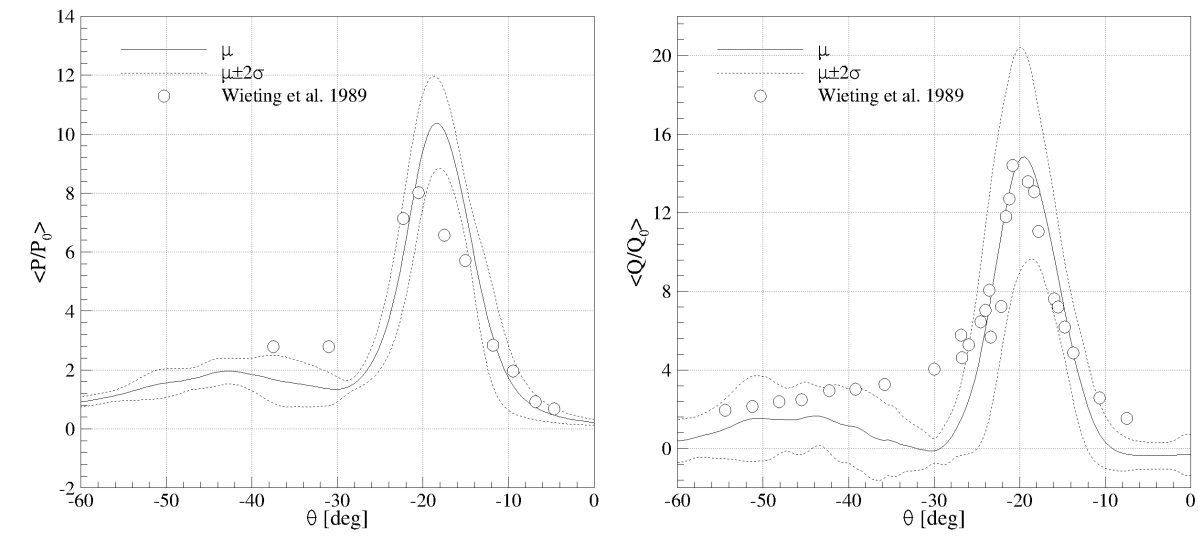

Figure 13: Edney IV pattern over a cylinder. Time averaged Normalized pressure profile (left), time averaged normalized heat flux (right). Thermally perfect gas model.

approach with methods based on polynomial curve fits is presented explicitly in terms of the $C_{P}$ and $C_{V}$ variation with the temperature only and not in terms of field variables. Some discrepancies are observed as the temperature increases due to effects that cannot be properly modeled by means of an harmonic oscillator and other coupling effects between vibration and rotation. Nevertheless, for temperatures below $3,000 \mathrm{~K}$ the proposed model provides results that in the case of air are in agreement with the results that would be obtained with the polynomial fit approach. Despite being presented in the case of the node-pair GKS scheme, the formulation is general to be adopted by any Boltzmann-BGK method.

One major recognized limiting factor of any GKS scheme is its computational demand resulting form the necessity to compute for each node-pair the moments of the particle distributions function for each conserved quantity. A marginal increase in computational cost was observed in the case of thermally perfect gas due to the necessity of computing the additional moments for the vibrational and electronic modes. In the context of gas-kinetic schemes, the adoption of the 
present approach will bring no advantages in terms of computational cost when compared to polynomial fits, but the increase in computational cost would in any case be moderate. High CPU demand is a known issue of all Boltzmanntype methods and such an aspect is the topic of ongoing research on parallel architectures and GPU processing.

\section{Acknowledgements}

The authors wish to acknowledge the support provided by the UK Space Agency and Lockheed Martin Corporation (Dr. N. Allen and Dr. L. Uribarri) thorough the National Space Technology Program (NSTP-PF026). The authors also wish to acknowledge the relevant comments and support of Prof. K. Xu from the Hong Kong University of Science and Technology. Results were obtained using the EPSRC funded ARCHIE-WeSt High Performance Computer (www.archie-west.ac.uk). EPSRC grant no. EP/K000586/1.

\section{References}

[1] G. A. Bird, Monte Carlo Simulation of Gas Flow, Ann. Rev. Flu. Mech. 10 (1979) 11.

[2] S. Succi, The lattice Boltzmann Equation for Fluid Dynamics and Beyond, Oxford University Press, 2001.

[3] R.-S. Myong, A generalized hydrodynamic computational model for rarefied and microscale diatomic gas flows, J. Comput. Phys. 195 (2004) 655-676.

[4] J. M. Reese, L. C. Woods, F. J. P. Thivet, S. M. Candel, A second-order description of shock structure, J. Comput. Phys. 117 (1995) 240.

[5] H. Grad, On the kinetic theory of rarefied gases, Commun. Pure Appl. Math. 2 (1949) 325. 
[6] R. K. Agarwal, K. Y. Yun, R. Balakrishnan, Beyond navier stokes: Burnett equations for flows in the continuum transition regime, Phys. Fluids 13

[7] S. Colonia, R. Steijl, G. Barakos, Kinetic models and gas-kinetic schemes for hybrid simulation of partially rarefied flows, AIAA Journal 54 (2016) 1264-1276.

[8] V. Titarev, Application of model kinetic equations to hypersonic rarefied

[9] Z. Guo, S. Shu, Lattice Boltzmann Method and its Applications in Engineering, World Scientific, 2013.

[10] D. A. Lockerby, J. M. Reese, M. A. Gallis, The usefulness of higher-order constitutive relations for describing the knudsen layer, Phys. Fluids 17

[11] K. Xu, K. H. Prendergast, Numerical Navier-Stokes solutions from gaskinetic theory, J. Comput. Phys. 114 (1994) 9-17.

[12] P. L. Bhatnagar, E. P. Gross, M. Krook, A model for collision processes in gases. i. small amplitude processes in charged and neutral one-component

[13] K. Xu, A gas-kinetic bgk scheme for the navierstokes equations and its connection with artificial dissipation and godunov method, J. Comput. Phys. 171 (1) (2001) $289-335$.

[14] K. Xu, L. Martinelli, A. Jameson, Gas-kinetic finite volume methods, flux vector splitting, and artificial diffusion, J. Comput. Phys 120 (1995) 48-65.

[15] K. Xu, J.-C. Huang, A unified gas-kinetic scheme for continuum and rarefied flows, J. Comput. Phys. 229 (2010) 7747-7764. 
[16] K. Xu, Z. Guo, Multitemperature gasdynamics equations for nonequilibrium flows, J. Comput. Math. 29 (5) (2011) 639-660.

[17] G. Cao, H. Liu, K. Xu, Physical modeling and numerical studies of three-dimensional non-equilibrium multi-temperature flows, Phys. Fluids $30(2018) 126104$.

[18] L. Pan, J. Cheng, S. Wang, K. Xu, A two-stage fourth-order gas-kinetic scheme for compressible multi-component flows, Commun. Comput. Phys. 22 (2017) 1123-1149.

[19] K. Xu, J.-C. Huang, An improved unified gas-kinetic scheme and the study of shock structures, IMA J. Applied Mathematics 76 (5) (2011) 698-711.

[20] M. Fossati, Numerical simulation of compressible flows in continuumtransitional regimes, Ph.D. thesis, Politecnico di Milano (2008).

[21] M. Fossati, A. Guardone, L. Vigevano, K. Xu, Kinetic node-pair formulation for two-dimensional flows from continuum to transitional regime, AIAA journal 51 (4) (2013) 784-796.

[22] H. Liu, K. Xu, A runge-kutta discontinuous galerkin method for viscous flow equations, J.Comput. Phys. 1 (2007) 1223-1242.

[23] H. Luo, L. Luo, K. Xu, A discontinuous galerkin method based on a bgk scheme for the navier-stokes equations on arbitrary grids, J.Comput. Phys. 1 (2009) 301-318.

[24] X. Ji, F. Zhao, W. Shyy, K. Xu, A familiy of high-order gas-kinetic schemes and its comparison with riemann solver based high-order methods, J. Comput. Phys. 356 (2018) 150-173. 
[25] M. Fossati, L. Vigevano, A node-pair bgk kinetic scheme for flows at moderate knudsen numbers, in: 6th European Symposium on Aerothermodynamics for Space Vehicles, January, 2008.

[26] L. Formaggia, V. Selmin, Unified construction of finite element and finite volume discretizations for compressible flow, Int. J. Num. Meth. Eng 39 (1996) 1-32.

[27] J. D. Anderson, Hypersonic and high temperature gas dynamics, AIAA Education Series, 2006.

[28] B. J. McBride, S. Gordon, M. A. Reno, Coefficients for calculating thermodynamic and transport properties of individual species, Tech. Rep. NASA TM-4513, National Aeronautics and Space Administration, Washington D.C. (1993).

[29] W. Li, M. Kaneda, K. Suga, An implicit gas kinetic bgk scheme for high temperature equilibrium gas flows on unstructured meshes, Comput. Fluids 93 (2014) $100-106$.

[30] Z. Jiang, C. Yan, J. Yu, F. Qu, L. Ma, Effective high-order solver with thermally perfect gas model for hypersonic heating prediction, Appl. Therm. Eng. 99 (2014) 147-159.

[31] V. Selmin, The node-centred finite volume approach: Bridge between finite differences and finite elements, Comput. Meths. Appl. Mech. 102 (1993) $107-138$.

[32] L. Formaggia, V. Selmin, Simulation of hypersonic flows on unstructured grids, Int. J. Num. Meth. Eng 114 (1994) 569-606.

[33] C. Cercignani, The Boltzmann Equation and its Applications, Springer650 Verlag, 1975. 
[34] S. Chapman, T. G. Cowling, The Mathematical Theory of Nonuniform Gases, Cambridge University Press, 1952.

[35] K. Xu, Regularization of the Chapman Enskog expansion and its description of shock structure, Phys.Fluids 14 (2002) L17-L20.

[36] W. G. Vincenti, C. H. Kruger, Introduction to physical gas dynamics, Introduction to physical gas dynamics, by Vincenti, Walter Guido; Kruger, Charles H. New York, Wiley [1965].

[37] Q. B. Li, S. Fu, K. Xu, Application of bgk scheme with kinetic boundary conditions in hypersonic flow, AIAA Journal 43 (2005) 2170-2176.

[38] K. Xu, Gas-kinetic schemes for unsteady compressible flow simulations, Vol. 3, Van Kareman Institute, 1998.

[39] M. Fossati, Evaluation of aerodynamic loads via reduced-order methodology, AIAA J. 53 (8) (2015) 2389-2405.

[40] T. D. Economon, F. Palacios, S. R. Copeland, T. W. Lukaczyk, J. J. Alonso, SU2: An open-source suite for multiphysics simulation and design, AIAA Journal 54 (3) (2015) 828-846.

[41] J. B. Scoggins, T. E. Magin, Development of Mutation ${ }^{++}$: Multicomponent Thermodynamic And Transport properties for IONized plasma written in $\mathrm{c}^{++}$, AIAA paper 2014-2966 53 (8) (2014) 2389-2405.

[42] W. Jones, J. Muylaert, P. Erbland, J. Schmisseur, Assessment of aerothermodynamic flight prediction tools through ground and flight experimentation, Tech. rep., NATO Science and Technology Organization, RTO-TRAVT-136 (2011).

[43] A. S. Durna, M. Barada, B. Celik, Shock interaction mechanisms on a 675 double wedge at Mach 7, Phys. Fluids 28 (2016) 096101. 
[44] A. B. Swantek, J. M. Austin, Flowfield establishment in hypervelocity shock-wave/boundary-layer interactions, AIAA J. 53 (2015) 311-320.

[45] M. J. Wright, K. Sinha, J. Olejniczak, G. V. Candler, T. D. Magruder, A. Smits, Numerical and experimental investigation of double-cone shock interactions, AIAA J. 32 (2000) 2268-2276.

[46] B. Edney, Anomalous heat transfer and pressure distributions on blunt bodies at hypersonic speeds in the presence of an imping shock, Tech. rep., FFA Report 115, Aero. Res. Institute of Sweden (1986).

[47] T. D. Magruder, An experimental study of shock/shock and shock/ boundary layer interactions on double-cone geometries in hypersonic flow, Ph.D. thesis, Princeton University (1997).

[48] X. Zhong, Application of essentially nonoscillatory schemes to unsteady hypersonic shock-shock interference heating problems, AIAA J. 32 (1994) $1606-1616$.

690

[49] A. R. Wieting, M. S. Holden, Experimental shock-wave interference heating on a cylinder at Mach 6 and 8, AIAA J. 27 (1989) 1557-1565.

[50] K. Xu, M. Mao, L. Tang, A multidimensional gas-kinetic bgk scheme for hypersonic viscous flow, J. Comput. Phys. 203 (2005) $405-421$. 